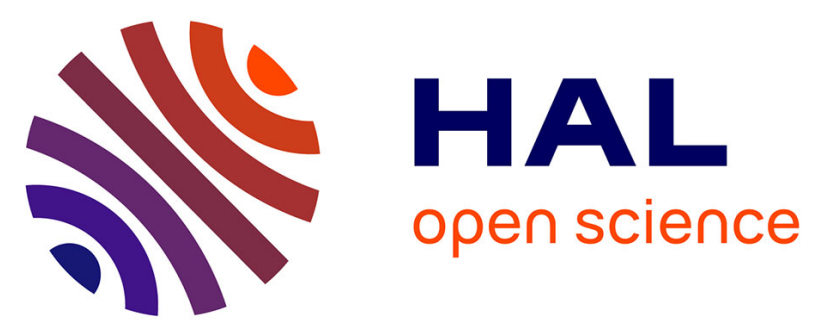

\title{
A 2D Markov chain for modelling powder mixing in alternately revolving static mixers of Sysmix $(R)$ type
}

Denis Ponomarev, Vadim Mizonov, Henri Berthiaux, Cendrine Gatumel, Janos Gyenis, Elena Barantseva

\section{- To cite this version:}

Denis Ponomarev, Vadim Mizonov, Henri Berthiaux, Cendrine Gatumel, Janos Gyenis, et al.. A 2D Markov chain for modelling powder mixing in alternately revolving static mixers of Sysmix (R) type. Chemical Engineering and Processing: Process Intensification, 2009, 48 (11-12), pp.1495-1505. 10.1016/j.cep.2009.10.004 . hal-01649516

\section{HAL Id: hal-01649516 https://hal.science/hal-01649516}

Submitted on 7 Nov 2019

HAL is a multi-disciplinary open access archive for the deposit and dissemination of scientific research documents, whether they are published or not. The documents may come from teaching and research institutions in France or abroad, or from public or private research centers.
L'archive ouverte pluridisciplinaire HAL, est destinée au dépôt et à la diffusion de documents scientifiques de niveau recherche, publiés ou non, émanant des établissements d'enseignement et de recherche français ou étrangers, des laboratoires publics ou privés. 


\title{
A 2D Markov chain for modelling powder mixing in alternately revolving static mixers of Sysmix ${ }^{\circledR}$ type
}

\author{
Denis Ponomarev a ${ }^{\mathrm{a}}$ Vadim Mizonov $^{\mathrm{a}}$, Henri Berthiaux ${ }^{\mathrm{b}, *}$, Cendrine Gatumel $^{\mathrm{b}}$, \\ Janos Gyenis ${ }^{\mathrm{c}}$, Elena Barantseva ${ }^{\mathrm{a}}$ \\ a Department of Applied Mathematics, ISPEU, Rabfakovskaya 34, 153003 Ivanovo, Russia \\ ${ }^{\mathrm{b}}$ RAPSODEE Centre, École des Mines d'Albi, FRE CNRS 3213, campus jarlard, route de Teillet, 81013 Albi, France \\ ${ }^{\mathrm{c}}$ RIChPE FIT, University of Pannonia, Egyetem u. 2, H-8200 Veszprem, Hungary
}

Keywords:

Static mixer

Mixing kinetics

Powder mixing

Markov chains

Stochastic process

\begin{abstract}
A B S T R A C T
A two-dimensional model of the flow and mixing of particulate solids has been developed on the basis of the Markov chain theory for an alternately revolving static mixer of Sysmix ${ }^{\circledR}$ type. In such a system, mixing occurs in both vertical and horizontal directions. Simulations are presented here to investigate the effect of the initial loading of the components, as well as the effect of the values of the transition probabilities that constitute the main parameters of the model. It is shown that a horizontal arrangement of the components always leads to better mixture quality and improved mixing kinetics. This research is presented for nonsegregating mixtures, as well as potentially segregating mixtures, for which the empirically well-known oscillations in variance are represented by the model. Results suggest that there is a rational way of approaching a static-mixing problem with regard to the initial loading of the component and the optimal number of revolutions. Comparison of model results with experimental data published previously for a Sysmix ${ }^{\circledR}$ apparatus contributes to validating the viability of the model.
\end{abstract}

\section{Introduction}

Choosing a powder mixer is a complex and time-consuming exercise for a chemical engineer. To select a simple distillation column, the engineer will probably spend several hours deciding on dimensions and characteristics, and a further day or more consulting vendors and purchasing the column. To select an appropriate powder mixer, the time required may be up to several months. This delay in the decision-making process can be explained by the lack of knowledge of powder characterization in general, and of powder rheology in particular. As a consequence, the range of technologies available for mixing powders is extremely large. The shape of a tumbler mixer may be a cylinder, a sphere, a cube, a double-cone, a double cylinder. The axis of rotation may differ from the axis of symmetry, and inserts can be included in the design. Convective mixers all differ from each other by the shape of the stirrer, which may be a ribbon, paddles mounted on a shaft, blades mounted on a frame, multiple screws, or a planetary motion with various axis of rotation-all this being combined with the different possible shapes of the mixing vessel. The broad category of "home-made" mixers,

\footnotetext{
* Corresponding author.

E-mail address: berthiau@enstimac.fr (H. Berthiaux).
}

of which container-mixers form a part, are subject to numerous failures that occur in scale-up and design procedures.

Depending on the manufacturing possibilities, such as the availability of spare parts, other alternatives like fluidised-bed mixers, static or silo mixers may be envisaged. In these types, the general motion of the particles is induced by gravity and the process operates by multiple passes or by the alternate revolving of the mixer. Insertions that are placed inside the vessel provoke dispersion of the particles (see Fig. 1). Static mixers provide a greater contact area between particles of different nature, which is a preliminary to the achievement of a finer-scale mixture. They could probably give good results as premix units, for example at the inlet of a continuous plough mixer, but have never been studied from this point of view.

Modelling of powder mixing and flow is a very valuable approach to the understanding of the collective and individual motion of particulates, and therefore may prove useful in improving mixer design. Distinct Element Models (DEMs) have been developed and applied to real mixing cases since the midnineteen nineties. DEMs are based on calculating particle-particle and particle-wall interactions, a procedure that requires the detection of the contacts between particles, which can be difficult if these are of non-spherical shape [1]. This procedure limits considerably the number of elements (particles) that need to be taken into account in the simulation and has led most researchers to develop 

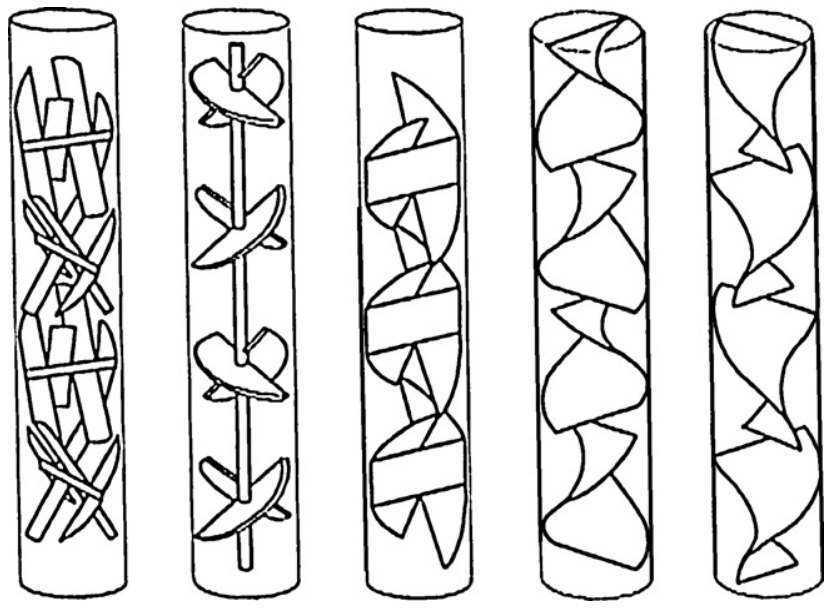

Fig. 1. Typical mixing element designs in static powder mixers.

hybrid models for which DEM can be considered as a potential "building block" (see [2] or [3]). The building into which such a block may be incorporated should be a meso or a macroscopic model, a category to which Markov chain models pertain.

Markovian models have been applied to a wide range of powder mixers since the beginning of the nineteen-seventies: they have been applied to static mixers in simple cases [4-6], to fluidised-bed mixers $[7,8]$, to tumbler mixers $[9,10]$, and to continuous convective mixers [11]. The models consist in representing the general flow pattern by a network of cells, the transition between these during fixed intervals of time being assimilated to conditional probabilities. The matrix representation of the model allows state occupation to be calculated rapidly and also facilitates the derivation of macroscopic criteria of process efficiency, such as mixture homogeneity. Recently, we developed a Markov chain model to simulate the evolution of the axial non-homogeneity of binary mixtures in static mixers of "tube type", accounting for particle segregation during downward gravity flow [12]. In essence, it is a one-dimensional cell model which is not able to capture the crosswise mixing of particles during their flow through the tube. In the present paper, we extend this model to a general two-dimensional flow representation, as occurring in a Sysmix ${ }^{\circledR}$ mixer. In particular, we will focus on the effect of initial feed loading, on the number of revolutions and on powder flow by its macroscopic transcription through Markovian transition probabilities. This analysis will be supported by numerical simulations to capture different possible outcomes of such processes. Comparison with published experimental data will also help to illustrate the viability of the model.

\section{Markov chain model formalism}

The Sysmix ${ }^{\circledR}$ was invented and studied in the MUKKI Chemical Engineering Institute in Veszprém (see [13-16]). It can be said to consist of an intermediate design between a static mixer and a tumbler mixer with inserts (see Fig. 2). The solid material is introduced in the lower part of the vessel, which is revolved by $180^{\circ}$ with respect to its horizontal axis of symmetry. After a standstill of
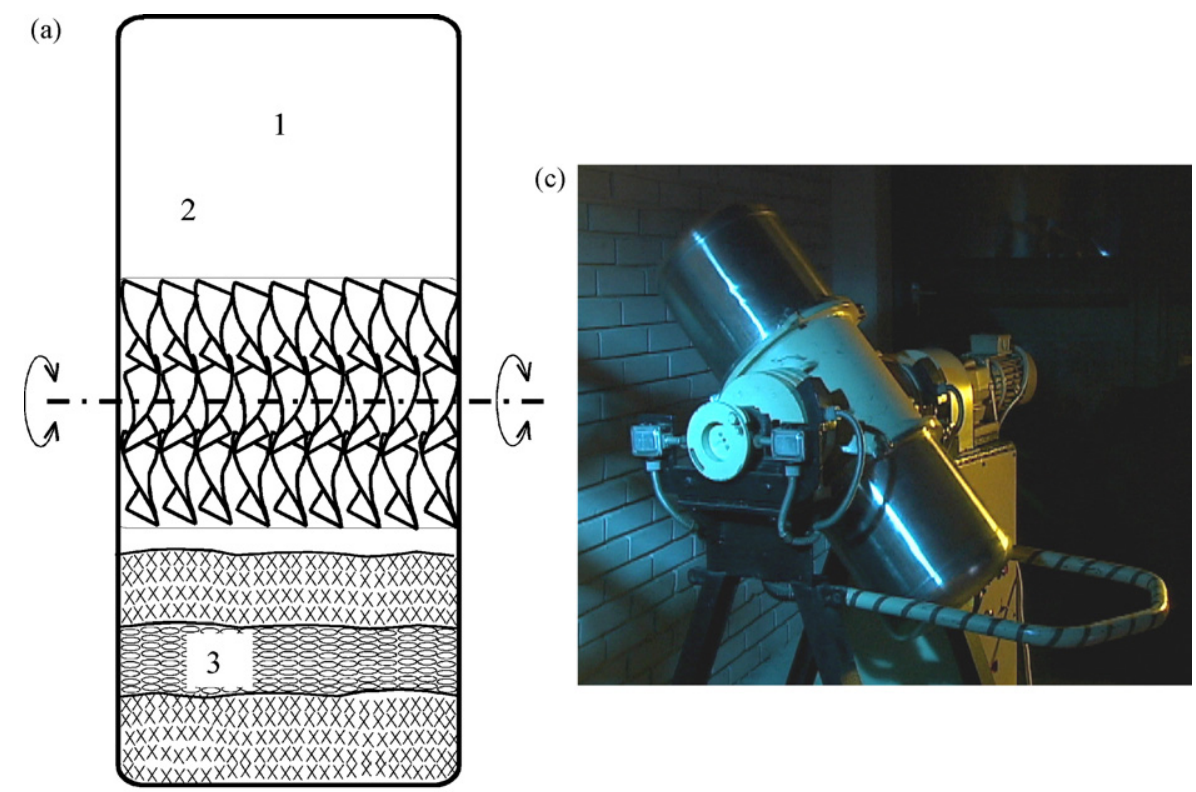

(b)

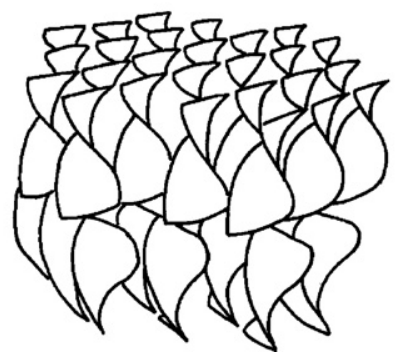

(d)

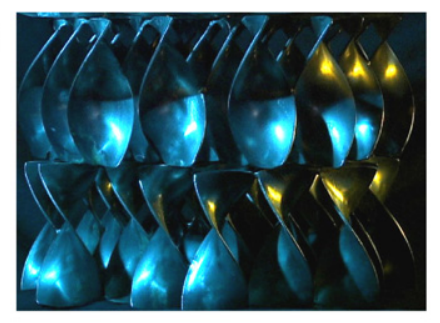

Fig. 2. Sysmix ${ }^{\circledR}$ bulk solids mixer. (a) Schematic diagram of the mixer (1: upper (feeder) container, 2: static mixer elements, 3: lower (receiver) container with the layered powder components to be mixed, 4: rotation shaft), (b) grids composed of helical static mixer elements, (c) photo of the mixer in operation, and (d) photo of the static mixers grid. 

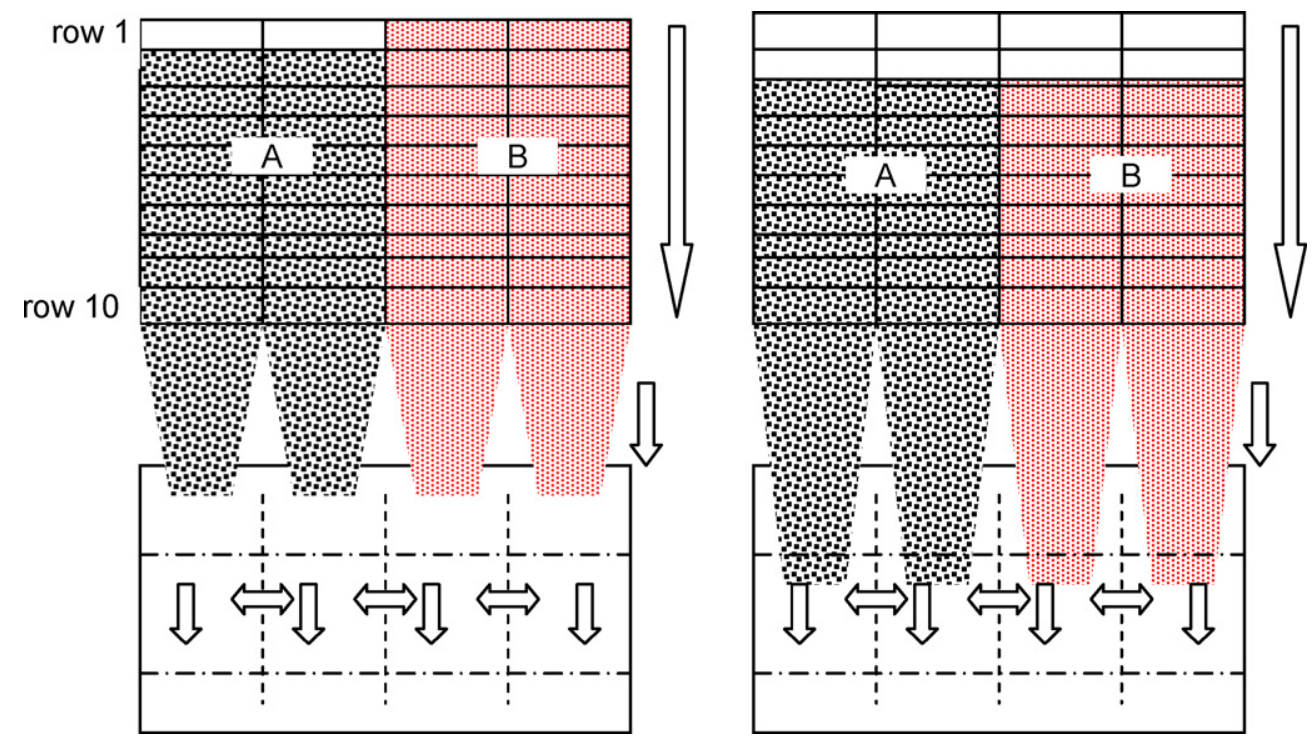

Fig. 3. Material injection from the feeding section to the first row of the mixing zone.

a few seconds to let the particles reach the bottom of the mixer, it is rotated in the opposite direction. Mixing continues by alternate rotation in this way. The mixer is filled up with mixing elements acting as packing in absorption or distillation columns, so as to increase and renew the contact area between different regions of the particle bed. In a certain manner, this idea is in contradiction with the need to let the particles flow easily through the mixer and avoid blockage and/or intermittent powder flow. Process efficiency is therefore a complex combination of mixer design (number, size, shape, spatial disposition of mixing elements, packing porosity, etc.), process operating conditions (feeding strategy, rotational speed, revolving frequency, number of revolutions), as well as powder flow characteristics as a response to a given mixer configuration. The model developed will therefore have to account for these aspects of the process, and in particular for the downward/crossing general particle flow.

\subsection{General approach to 2D flow modelling}

The Markov chain modelling of particulate flow consists in representing the process by a network of cells through which particles can transit, defining transition probabilities between the cells (or state) and summarizing the whole procedure in matrix notation with appropriate rules. The knowledge of state occupation during the operation allows important process characteristics to be calculated, such as Residence Time Distribution (RTD) for continuous mixers or mixture homogeneity. Readers should refer to our previous articles to have a complete view of the model description, the modelling strategies, and the various existing or potential applications [17-19].

In a static mixer of Sysmix ${ }^{\circledR}$ type, particle flow and mixing is of a sequential nature, as the revolution procedure is timediscontinuous. The model will therefore consist of three basic "blocks":

1. feeding of material from the upper container to the mixing zone;

2. motion of material through the mixing zone;

3. re-collection of the "micro" portions, which have come from the mixing zone, into constant sample volumes.

Material feed to the mixing zone is organized by means of a special feeder or loading container. The latter is represented by the two-dimensional cell space where the number of cells is equal to the number of columns of mixing elements and the number of rows equal to the number of samples chosen in a vertical direction. Let us consider a binary mixture case (A/B). In an initial approach, component $A$ is placed in the left-hand part of the feeder (upper container) and component $B$ is placed in its right-hand part. Material injection into the mixing zone represented by four columns is shown in Fig. 3. Material in the loading container is formally separated into columns of the array modelling the mixing zone, and into the layers corresponding to the particle mass flow rate and the chosen transition duration $\Delta t$ (10 layers in the illustration). At each transition, one layer enters the upper row of cells representing the mixing zone and brings with it a certain mass of a key component, which is under observation. Thus there is a continuous feed to the mixing zone during several first transitions, the number of which is equal to the chosen number of layers. However, the outflow into the corresponding absorbing container at the bottom caused by each inflow during one transition will be distributed over transitions and will form different layers in the absorbing container. Note that only the total mass of material should be equal in both containers after passage of the material through the mixing zone is finished. In order to model the recurring procedure of passages, the mass of the key component in the absorbing container is to be re-collected into the same layers as in the loading container.

We now focus on the mixing zone and illustrate the strategy of building a so-called "2D-model" by considering the simple case of a $3 \times 3$ two-dimensional network of cells as shown in Fig. 4 . The cells are considered to occupy identical volumes in the mixer, but

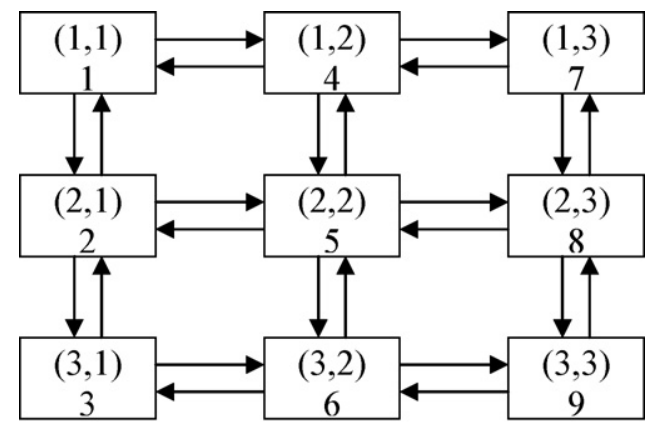

Fig. 4. Example of a $3 \times 32$ D Markov chain cell model 
the corresponding particle masses may be different. The particles of a certain component $\mathrm{A}$ of a binary mixture can occupy the cell " $i j$ " with a frequency, or a concentration, $s_{i j}$. Consequently, the state probability distribution along cells can be defined by the matrix $\mathbf{S}_{m}$ :

$\boldsymbol{S}_{m}=\left[\begin{array}{lll}s_{11} & s_{12} & s_{13} \\ s_{21} & s_{22} & s_{23} \\ s_{31} & s_{32} & s_{33}\end{array}\right]$

In order to perform the next matrix operations, model cells should be renumbered in a sequence shown in Fig. 4:

$\boldsymbol{S}_{m}=\left[\begin{array}{lll}s_{1} & s_{4} & s_{7} \\ s_{2} & s_{5} & s_{8} \\ s_{3} & s_{6} & s_{9}\end{array}\right]$

which should in turn be transformed into the state vector:

$\boldsymbol{S}=\left[\begin{array}{c}s_{1} \\ s_{2} \\ \ldots \\ s_{8} \\ s_{9}\end{array}\right]$

The mixing zone is represented by a matrix of transition probabilities of size $9 \times 9$, since mixing occurs in two directions, vertical and horizontal. Each column contains transition probabilities from the given cell to the neighbouring cells according to its direct unary numeration. The main diagonal is concerned with probabilities of remaining in cells during one transition. These probabilities can be obtained by subtracting the sum of transition probabilities to the neighbouring cells from 1 . The probabilities of such transitions should be placed in the columns of the matrix:

$\boldsymbol{P}=\left[\begin{array}{ccccccccc}1 \rightarrow 1 & 2 \rightarrow 1 & 0 & 4 \rightarrow 1 & 0 & 0 & 0 & 0 & 0 \\ 1 \rightarrow 2 & 2 \rightarrow 2 & 3 \rightarrow 2 & 0 & 5 \rightarrow 1 & 0 & 0 & 0 & 0 \\ 0 & 2 \rightarrow 3 & 3 \rightarrow 3 & 0 & 0 & 6 \rightarrow 3 & 0 & 0 & 0 \\ 1 \rightarrow 4 & 0 & 0 & 4 \rightarrow 4 & 5 \rightarrow 4 & 0 & 7 \rightarrow 4 & 0 & 0 \\ 0 & 2 \rightarrow 5 & 0 & 4 \rightarrow 5 & 5 \rightarrow 5 & 6 \rightarrow 5 & 0 & 8 \rightarrow 5 & 0 \\ 0 & 0 & 3 \rightarrow 6 & 0 & 5 \rightarrow 6 & 6 \rightarrow 6 & 0 & 0 & 9 \rightarrow 6 \\ 0 & 0 & 0 & 4 \rightarrow 7 & 0 & 0 & 7 \rightarrow 7 & 8 \rightarrow 7 & 0 \\ 0 & 0 & 0 & 0 & 5 \rightarrow 8 & 0 & 7 \rightarrow 8 & 8 \rightarrow 8 & 9 \rightarrow 8 \\ 0 & 0 & 0 & 0 & 0 & 6 \rightarrow 9 & 0 & 8 \rightarrow 9 & 9 \rightarrow 9\end{array}\right]$

Let us consider the mixing zone of a Sysmix ${ }^{\circledR}$-type mixer in more detail. It is made of mixing elements, axially installed inside the mixer. Each element is of helical shape and is slewed around its vertical axis by $90^{\circ}$ relative to the previous and subsequent elements placed above and below the element in question. Consequently, material flow will occur along the two sides of each mixing element so that the material goes to the bottom of this element and to other neighbouring zones. A cell model of the process is represented in Fig. 5. Each cell represents one side of a mixing element, the mixing effect happening in an axial direction at the interfaces between the successive cells. A vertical series of helical mixing elements is therefore represented by two columns of cells in the model. Crosswise mixing arises in the mixer because of particle rebound or interaction with the mixing elements. In the model, this is taken into account by only allowing transitions in the horizontal direction between the cells that are not separated by an element.

Finally, material is collected in the lower container, whose dimensions are identical to those of the upper container. Particles of each component arrive in the lower part of the vessel after the revolution of the mixer during the standstill period. It is therefore filled according to the flow behaviour of each component in the mixing zone. Each column of the lower container may then contain a different powder volume, and each cell may exhibit different concentrations in a specific component as long as mixture is not achieved at this scale of scrutiny. As the mixing procedure operates by turning the whole mixer body around its horizontal axis by $180^{\circ}$, the lower container serves as a feed for the next passage being turned upside down. The process can then be repeated and its dynamics can be followed by the Markov chain representation with appropriate rules of calculation.

\subsection{Transition matrix and process dynamics}

The dynamics of material motion through the mixing zone is defined by the following matrix equation:

$\boldsymbol{S}^{k+1}=\boldsymbol{P}\left(\boldsymbol{S}^{k}+\boldsymbol{S}_{f}^{(k)}\right)$

In the above equation, $\mathbf{P}$ is the transition matrix describing the exchanges of material in the mixing zone, $\mathbf{S}$ is the state column vector for the mixing zone and $\boldsymbol{S}_{f}^{(k)}$ is a column vector representing material feed. The latter can represent a step feed into the first row of the model as in the first pass of the present case, but can also represent a slot-type feed, a sinusoidal feed, a staged feed along the mixer's length and so forth. For a mixing zone consisting of a single column, this vector would be of size $n \times 1$. For $m$ mixing columns, its size is $n \times m$ and it is built by placing the feed vectors to each column one under another. The matrix of transition probabilities $\mathbf{P}$ is a block matrix, and has the following form according to the model scheme presented:

$$
\boldsymbol{P}=\left[\begin{array}{cccccc}
\boldsymbol{P}_{11} & \boldsymbol{P}_{12} & \boldsymbol{Z} & \boldsymbol{Z} & \boldsymbol{Z} & \boldsymbol{Z} \\
\boldsymbol{P}_{21} & \boldsymbol{P}_{22} & \boldsymbol{P}_{23} & \boldsymbol{Z} & \boldsymbol{Z} & \boldsymbol{Z} \\
\boldsymbol{Z} & \boldsymbol{P}_{32} & \boldsymbol{P}_{33} & \boldsymbol{P}_{34} & \boldsymbol{Z} & \boldsymbol{Z} \\
\boldsymbol{Z} & \boldsymbol{Z} & \boldsymbol{P}_{43} & \boldsymbol{P}_{44} & \boldsymbol{P}_{45} & \boldsymbol{Z} \\
\boldsymbol{Z} & \boldsymbol{Z} & \boldsymbol{Z} & \boldsymbol{P}_{54} & \boldsymbol{P}_{55} & \boldsymbol{P}_{56} \\
\boldsymbol{Z} & \boldsymbol{Z} & \boldsymbol{Z} & \boldsymbol{Z} & \boldsymbol{P}_{65} & \boldsymbol{P}_{66}
\end{array}\right]
$$

The matrices $\mathbf{P}_{i j}$, placed above the main diagonal of $\mathbf{P}$ are responsible for material motion from the right columns to the left ones, i.e., from the $j$ th columns to the $(j-1)$ th as only crosswise flow exists between adjacent elements. Similarly, the matrices $\mathbf{P}_{j i}$ placed below the main diagonal are responsible for material motion from the left columns to the right ones, i.e., from the $j$ th columns to the $(j+1)$ th. Matrices $\mathbf{P}_{i i}$ represent particle flow "inside" the $i$ th column, and $\mathbf{Z}$ is a zero matrix of size $4 \times 4$. It should be noted that the indexing of the sub-matrices in the above does not coincide with the indexing of mixing cells in the model.

The matrix of transition probabilities for the vertical helical mixing sets of Fig. 5 can be seen in Fig. 6 in accordance with the above remarks. If the probability to stay in a cell $p_{\mathrm{s}}$ is the same for a component whichever cell is considered, if $p_{\mathrm{c}}$ is the probability to transit in the horizontal direction, and $p_{\mathrm{d}}$ is the probability to transit in the vertical direction, the transition matrix contains three unknown parameters. It is also necessary to note that the matrix $\mathbf{P}$ has to be normalized, so that the sum of probabilities within each column of the matrix written in numbers is to be equal to 1 :

$$
\sum_{i=1}^{24} p_{i j}=1, \quad j=1,2, \ldots, 4
$$

It follows from (Eq. (7)) that $p_{\mathrm{d}}=\left(1-p_{\mathrm{c}}-p_{\mathrm{s}}\right) / 2$ is true for all matrices except $\mathbf{P}_{11}$ and $\mathbf{P}_{66}$; and $p_{\mathrm{d}}=\left(1-p_{\mathrm{s}}\right) / 2$ for the matrices $\mathbf{P}_{11}$ and $\mathbf{P}_{66}$. As a consequence, the problem is reduced to only two unknown parameters per component.

When all the particles have arrived at the bottom of the mixer, and been re-collected into the same layers as for the loading container, the obtained vector is rotated upside down and used as the feed vector for the next pass. Then the procedure repeats so that, from a process viewpoint, the mixer appears as a continuous mixer with a feed acting within a limited time interval. The transition matrix is a square matrix whose size must be chosen according to the number of mixing elements installed in the mixing zone. For 


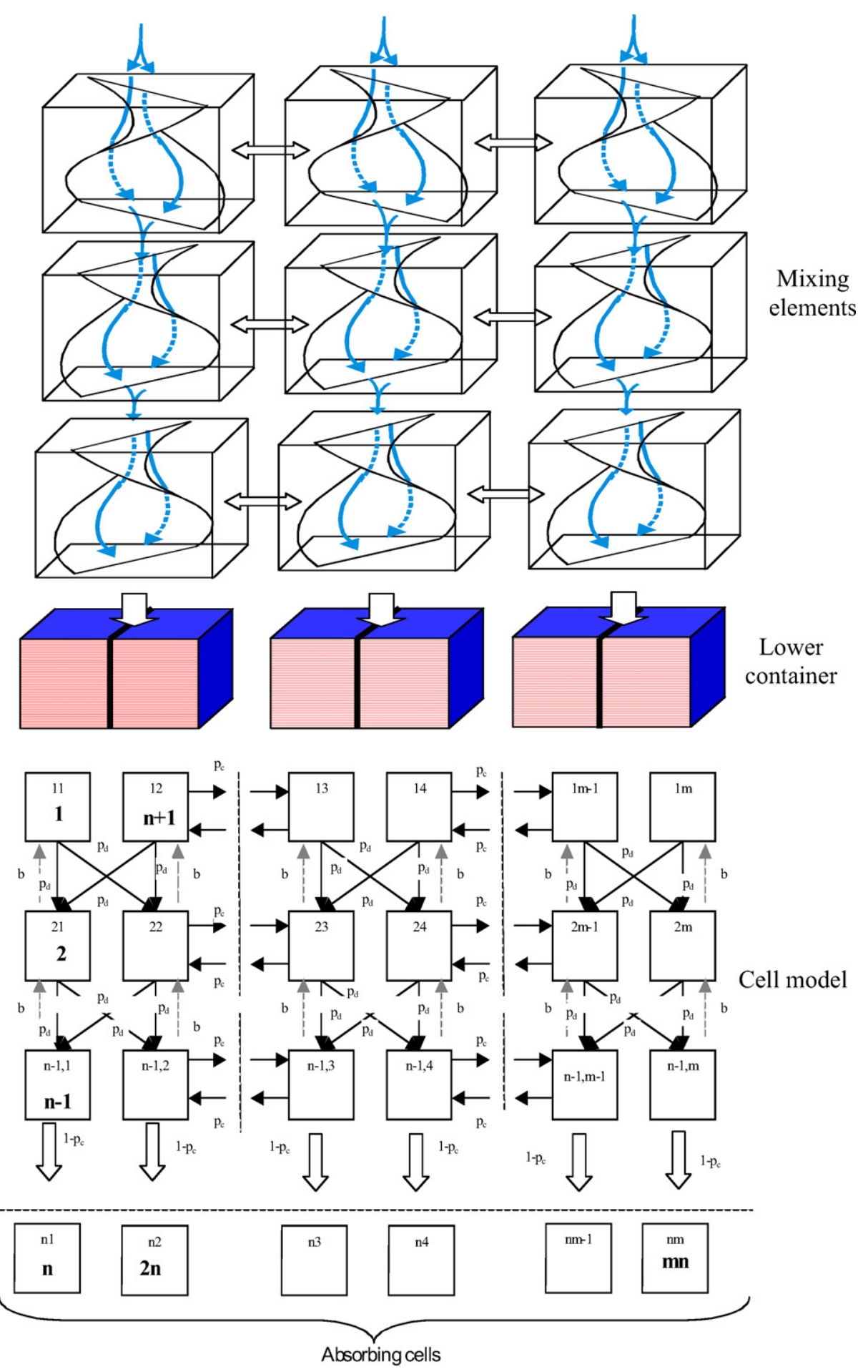

Fig. 5. Powder flow in a Sysmix ${ }^{\circledR}$-type static mixer (top) and the corresponding Markov chain model (bottom).

example, if the mixing section is made of 3 vertical arrangements of 4 mixing elements each, the size of the transition matrix will be $30 \times 30$ (taking into account the 6 absorbing states). However, as long as the transition probabilities are identical for each cell, this will not increase the number of possibly unknown parameters, nor affect the form of the block matrices, and the calculation time for simulating the dynamics of the process will be practically unaffected.

The above protocol is suitable to represent the flow of each component in the mixture. But if these components do not exhibit the same transition probabilities in the mixing zone, one must consider as many transition matrices as there are different component flow behaviours, at least in an initial approach. For instance, in the binary mixture case $\mathrm{A} / \mathrm{B}$, there will be four model parameters $p_{\mathrm{sA}}, p_{\mathrm{sB}}, p_{\mathrm{cA}}$, and $p_{\mathrm{CB}}$ that depend on the flow behaviour of the components inside the mixer. Finding relationships between bulk powder properties and element design with transition probabilities is a key issue here, but it remains outside of the scope of the present work. If the matrices $\mathbf{P}_{\mathrm{A}}$ and $\mathbf{P}_{\mathrm{B}}$ are the same for both components, their distribution in the lower container will asymptotically tend to be homogeneous, 


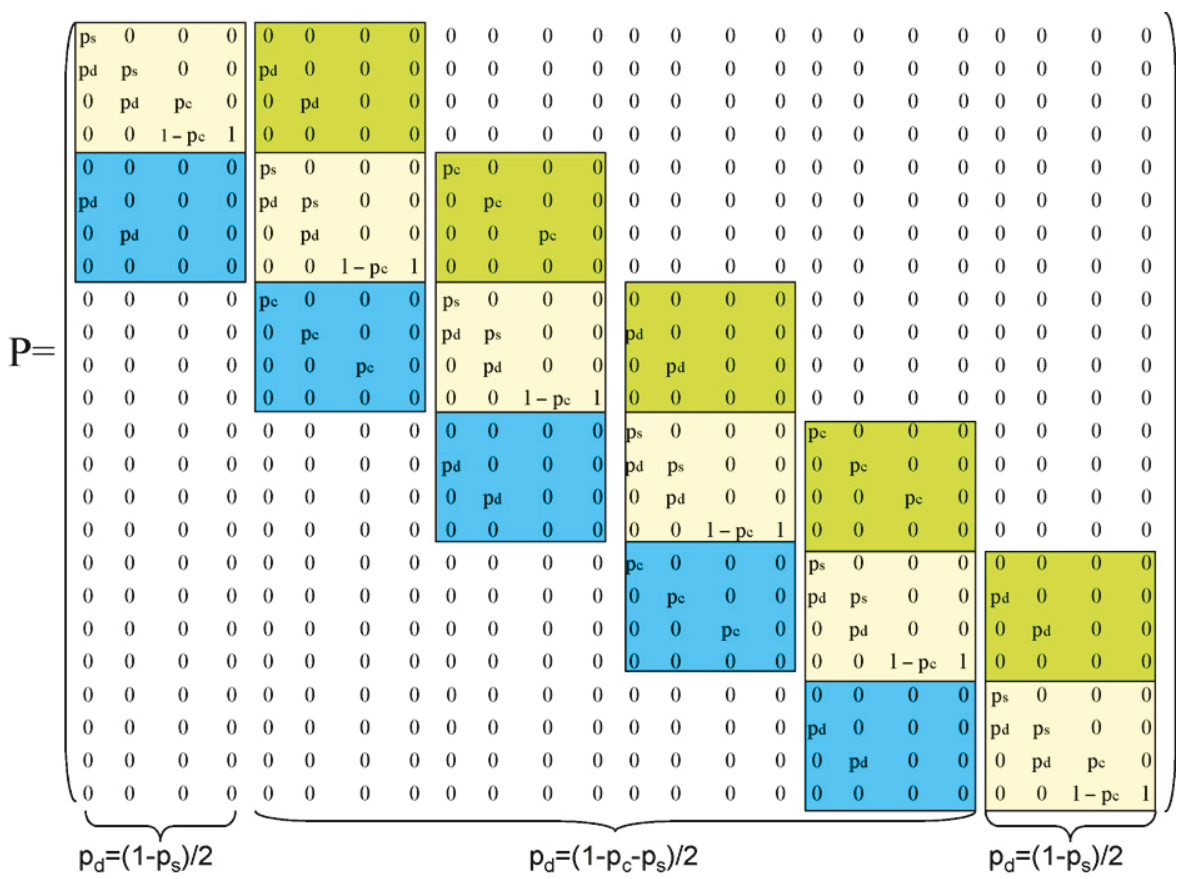

Fig. 6. Transition matrix related to the model presented in Fig. 5.

and thus the process optimization problem may consist in finding the number of revolutions of the mixer needed to guarantee the required mixture quality.

With respect to the previously published work mentioned above, the present model has a major advantage. In these previous models, one transition corresponded to one passage in the mixer, irrespective of the flow structure in the vessel. This is the reason why, despite their pioneering character, these studies could hardly be directly related to mixer design. In contrast, the 2D Markov model presented here represents flow at a mesoscopic level, that of the mixing elements. If one of these elements is removed, or replaced by another of a different shape, this will change the transition probabilities and affect particle flow dynamics. Applying the 2D modelling approach proposed here can therefore increase the predictability of mixer behaviour, thus improving tools for mixer design.

\subsection{Mixture homogeneity}

The degree of homogeneity of a powder mixture is the key characteristic that needs to be controlled in a mixing process. But the first step is to define the scale at which the mixture will be judged. In the present case, we will consider this "scale of scrutiny" as being that of the cells in the collector. For example, it will represent $1 / 40$ th of the total mixture in Fig. 3. Then, one has to define the component for which homogeneity will be calculated or evaluated (if sampling is necessary), generally the lowest-dosed ingredient or a key ingredient such as an active product. This is relatively easy to perform for the case of a binary mixture, but it becomes tedious for multi-component mixtures with various key ingredients as is very often the case in the pharmaceutical industry. The homogeneity of a mixture containing $N$ times the scale of scrutiny is also called the intensity of segregation and is usually quantified by the variance of the composition $c_{i}$ in sample $i$, for a specified key ingredient, over the $N$ possible samples of mean value $\langle c\rangle$ :

$\sigma^{2}=\frac{1}{N} \sum_{i=1}^{N}\left(c_{i}-\langle c\rangle\right)^{2}$
In the present case, if there are $m$ columns and $n$ rows in the feeding container, the above equation reads:

$\sigma^{2}=\frac{1}{m \cdot n} \sum_{i=1}^{n} \sum_{j=1}^{m}\left(c_{i j}-\langle c\rangle\right)^{2}$

It may also be interesting to calculate the variances in the horizontal and vertical directions, respectively:

$$
\begin{aligned}
& \sigma_{x}^{2}=\frac{1}{m} \sum_{j=1}^{m}\left(\frac{\sum_{i=1}^{n} c_{i j}}{n}-\left\langle c_{j}\right\rangle\right)^{2} \\
& \text { or } \sigma_{z}^{2}=\frac{1}{n} \sum_{i=1}^{n}\left(\frac{\sum_{j=1}^{m} c_{i j}}{m}-\left\langle c_{i}\right\rangle\right)^{2}
\end{aligned}
$$

In the above, $\left\langle c_{j}\right\rangle$ and $\left\langle c_{i}\right\rangle$ are mean concentrations in columns and rows, respectively. The coefficient of variation $\mathrm{CV}$ is usually employed to compare the actual homogeneity value to a standard. A typical criterion retained for drug liberation is $\mathrm{CV}<6 \%$, and will be considered as a reference in the present study:

$\mathrm{CV}=\frac{\sigma}{\langle c\rangle}$

In the model, the state vector directly represents the distribution of one of the components in the feeding container. As a consequence, the $\mathrm{CV}$ can easily be derived from the state vectors obtained after each passage in the mixer, and the time-change of these can provide a visual support to the progress of the mixing process.

\section{Some numerical simulations}

\subsection{Non-segregating mixtures}

When transition probabilities are the same for both components $A$ and $B$, obviously no segregation can take place and the asymptotical value of the variance is zero. However, the way this value is approached depends on the transition probabilities and on the initial arrangement of the components. This point is well known in 
(a)

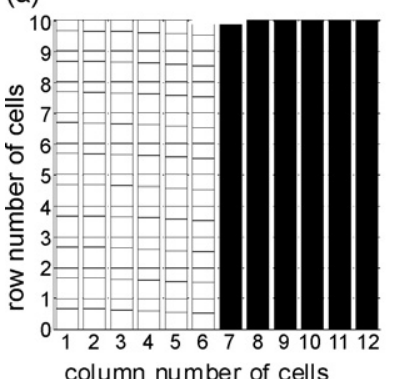

(b)

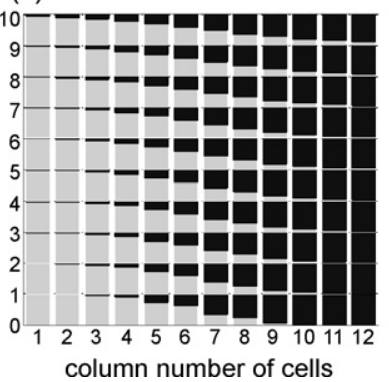

(c)

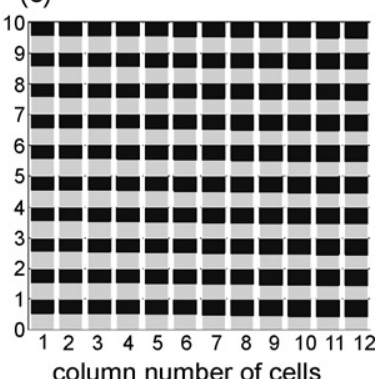

Fig. 7. State vector obtained for $p_{\mathrm{s}}=0.1$ and $p_{\mathrm{c}}=0.3$ (a, initial distribution; $\mathrm{b}$, distribution after one pass; $\mathrm{c}$, distribution after 10 passes).

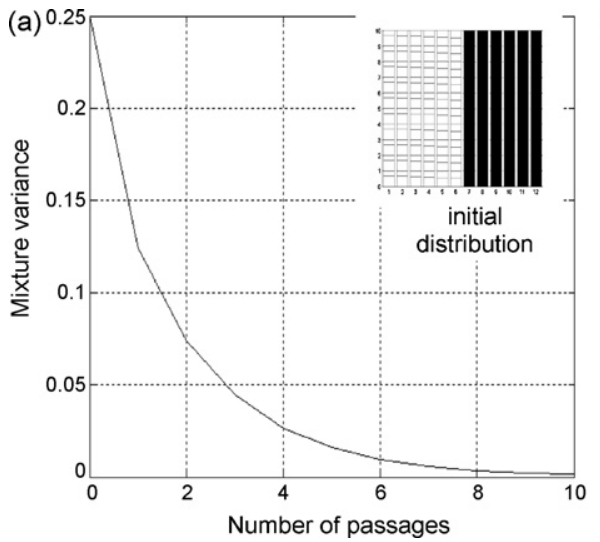

(b)

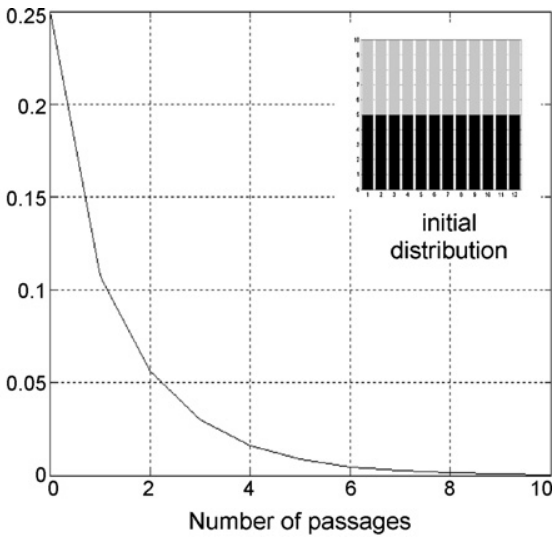

Fig. 8. Kinetics of binary mixing simulated with equal probabilities of the components $\left(p_{\mathrm{s}}=0.1 ; p_{\mathrm{c}}=0.3\right)$ as a function of the initial arrangement (vertical, a; horizontal, $\mathrm{b}$ ).

industrial practice, but has never been taken into account in a flow model for powders.

Fig. 7 shows the distribution of the material in the loading container after one pass in the mixing zone, as well as after 10 passes, for a vertical initial arrangement of the components, and for the following transition values: $p_{\mathrm{SA}}=p_{\mathrm{sB}}=0.1, p_{\mathrm{CA}}=p_{\mathrm{CB}}=0.3$. This diagram shows a concentration distribution among the cells in two directions and gives a visual illustration of the progress of the mixing process. The surface is horizontal and all sections are filled to the maximum because the probabilities for $A$ and $B$ are equal. We can note that the mixture is almost homogeneous at the scale considered (1/120th of the total mixture) after 10 mixer revolutions only. Fig. 8a shows the time-evolution of the variance for this vertical initial arrangement and allows the results to be compared with a horizontal initial distribution (Fig. 8b). Both curves monotonously decrease with the increase in the number of revolutions of the mixer, illustrating the absence of segregation phenomena. When the components are placed in two horizontal layers, the variance decreases more rapidly than in the case of the vertical configuration. For example, after two rotations, for the probabilities considered, the values of the variance are 0.06 and 0.075 for the horizontal and vertical cases, respectively.

To better illustrate this idea, a brief analysis was performed to test the sensitivity of the model to its parameters in the case of equal transition probabilities of the components. For this purpose, we considered a target homogeneity of $C V=6 \%$ and tried to find the combination of transition probabilities that allowed this value to be obtained. The results are reported in Fig. 9a (vertical arrangement) and Fig. 9b (horizontal arrangement).

In the vertical arrangement, low values of $p_{c}$ are to be coupled by very high values of $p_{\mathrm{s}}$ to give an acceptable homogeneity in a reasonable number of passes. In other words, a deficit in horizontal mobility must be compensated by the same characteristics in the vertical direction to prevent plug flow behaviour. For $p_{\mathrm{c}}$ values
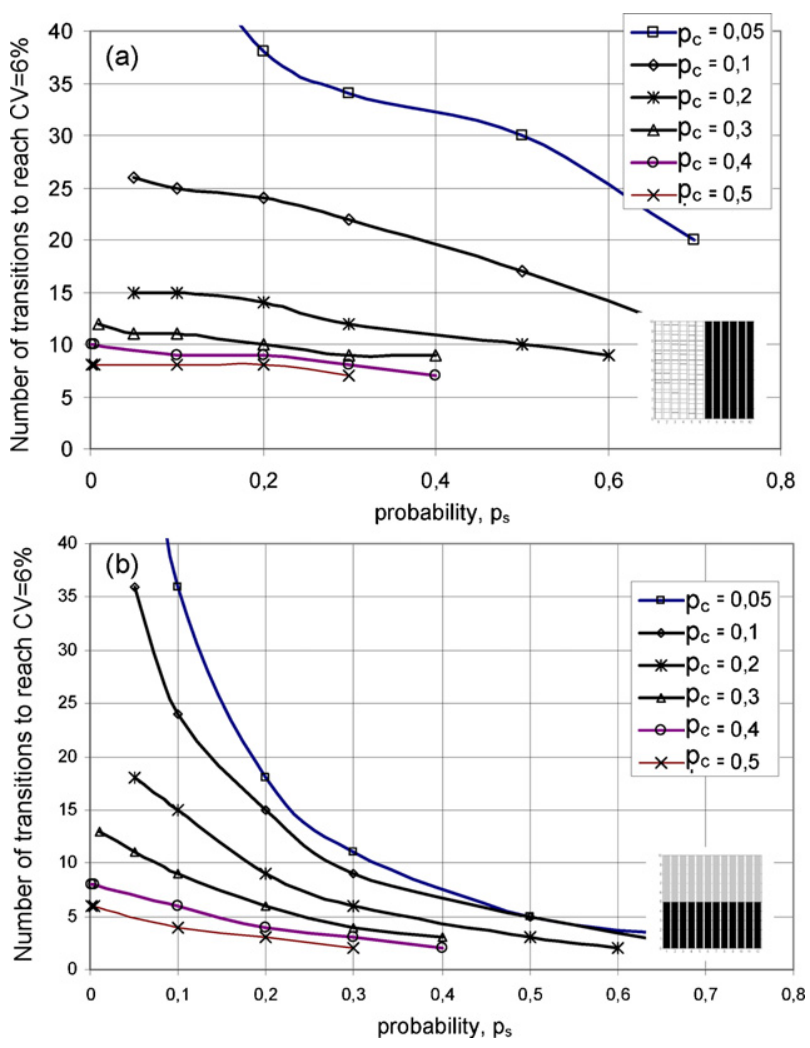

Fig. 9. Possible combinations of transition probabilities to reach a target value of $6 \%$ on the $\mathrm{CV}$ of the mixtures, as a function of the initial arrangement (vertical, a; horizontal, b). Both components have same transition probabilities. 

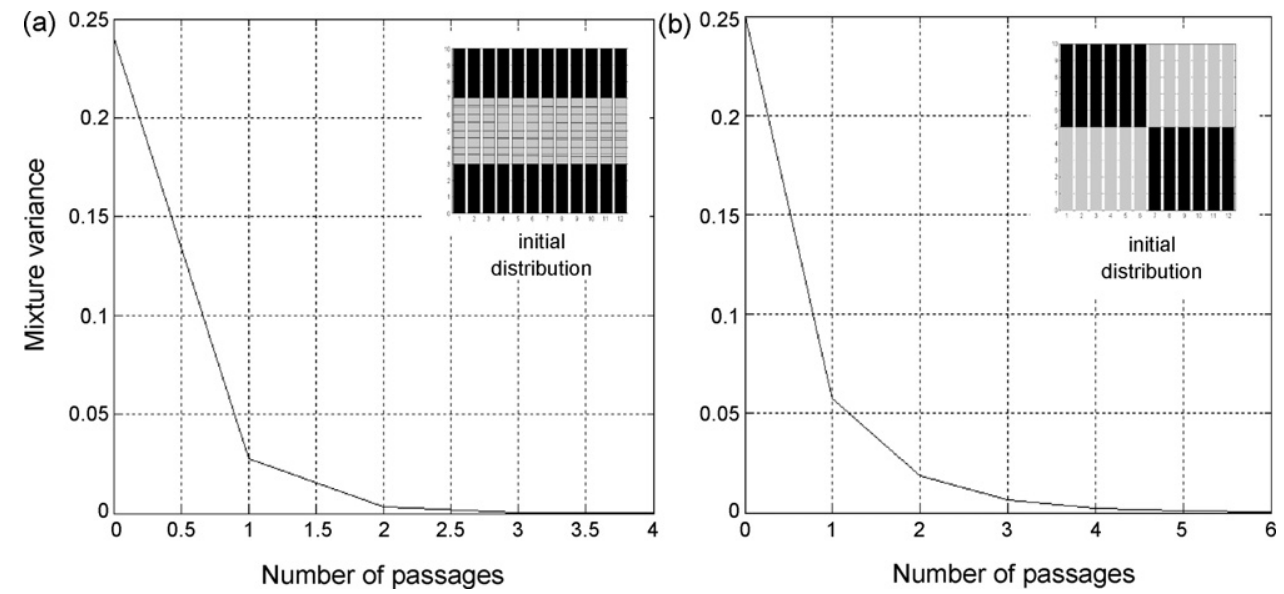

Fig. 10. Kinetics of mixing simulated with equal probabilities of the components $\left(p_{\mathrm{s}}=0.1 ; p_{\mathrm{c}}=0.3\right)$ with different initial scales of segregation (three horizontal layers, a; four blocks, b).
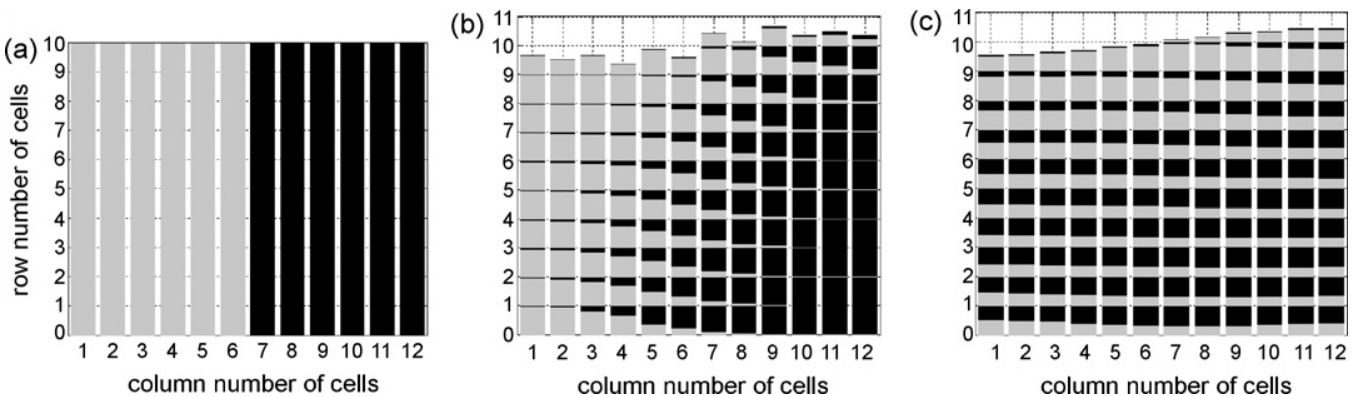

Fig. 11. State vector obtained for $p_{\mathrm{SA}}=p_{\mathrm{sB}}=0.1, p_{\mathrm{cA}}=0.5$, and $p_{\mathrm{sB}}=0.3$ (a, initial distribution; b, distribution after one pass; $\mathrm{c}$, distribution after eight passes).
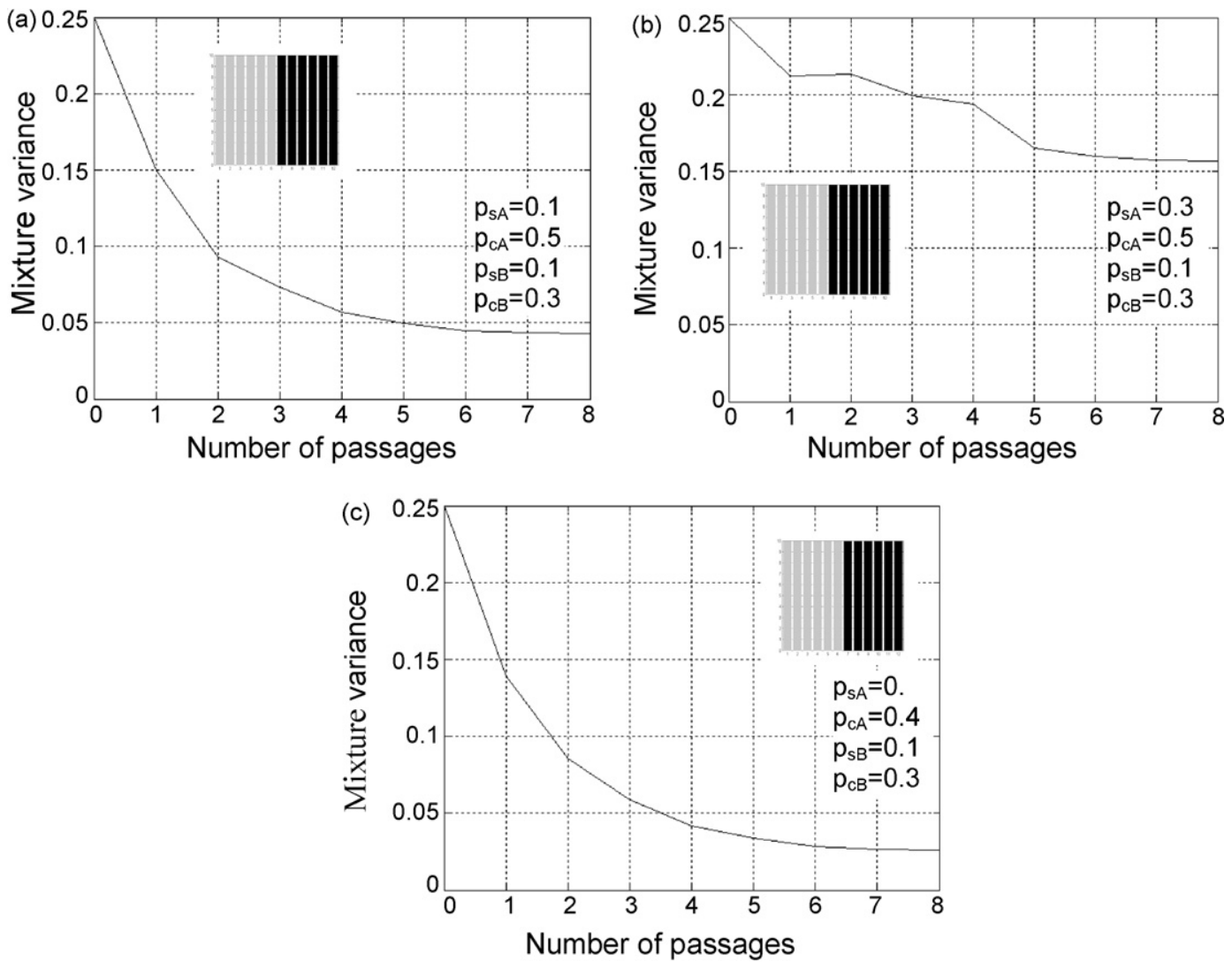

Fig. 12. Influence of transition probabilities on mixing kinetics for vertical arrangement of the components (A is figured in black). 

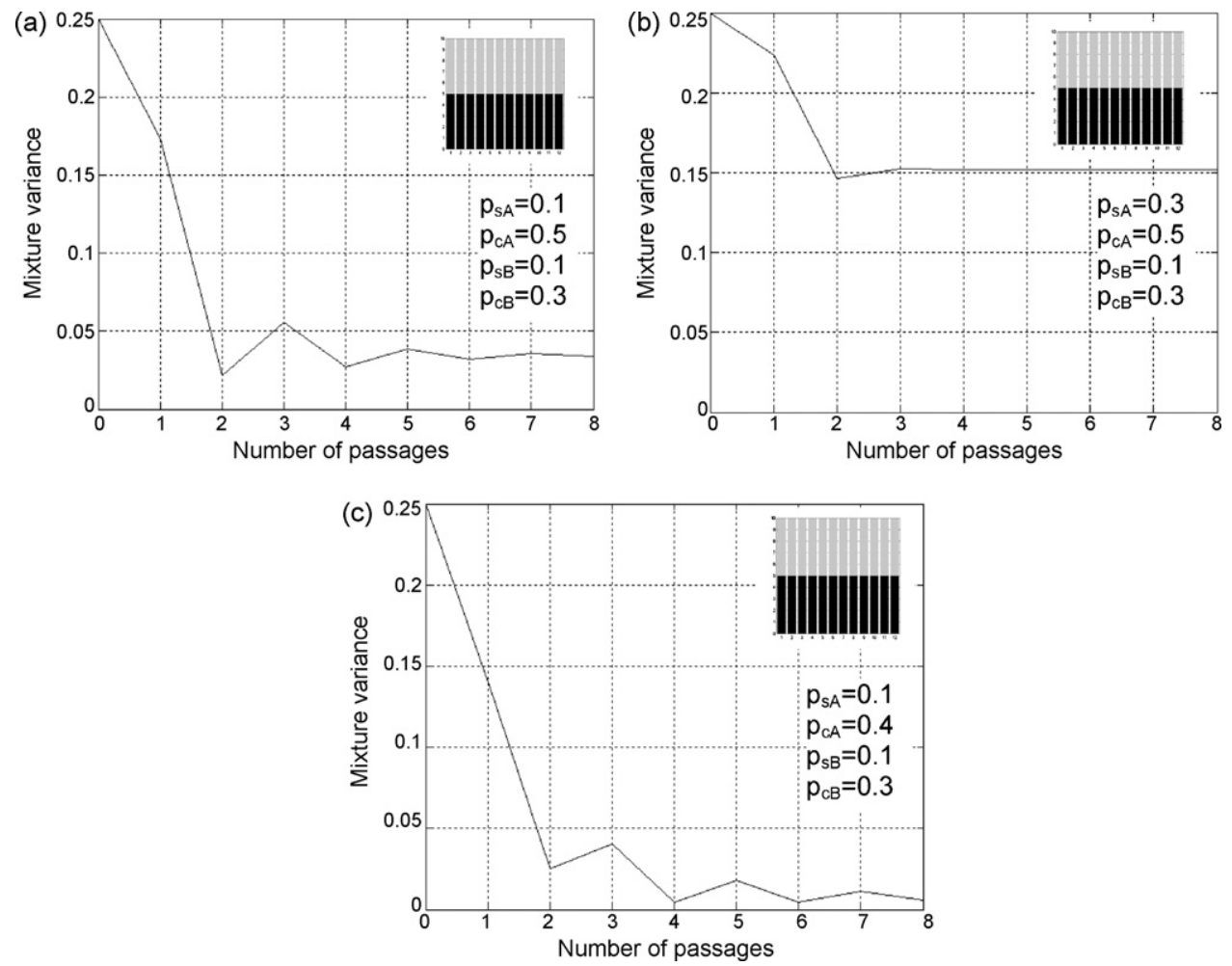

Fig. 13. Influence of transition probabilities on mixing kinetics for horizontal arrangement of the components (A is figured in black).

above 0.3 , the efficiency of the process becomes irrelevant of the $p_{\mathrm{s}}$ value, which means that once the horizontal mobility is ensured, the process is not sensitive to the vertical flow. The same conclusions may be drawn from the analysis of the results concerning the horizontal disposition, but the picture is globally less magnified for low values of $p_{c}$. High horizontal mobility is important but, even in this case, the vertical mobility is still influential, and must be low to "allow enough time" for mixing. For a given couple of transition probabilities, the target homogeneity will be attained with less transition (or revolution) if the initial arrangement of the components is horizontal rather than vertical. This is all the more

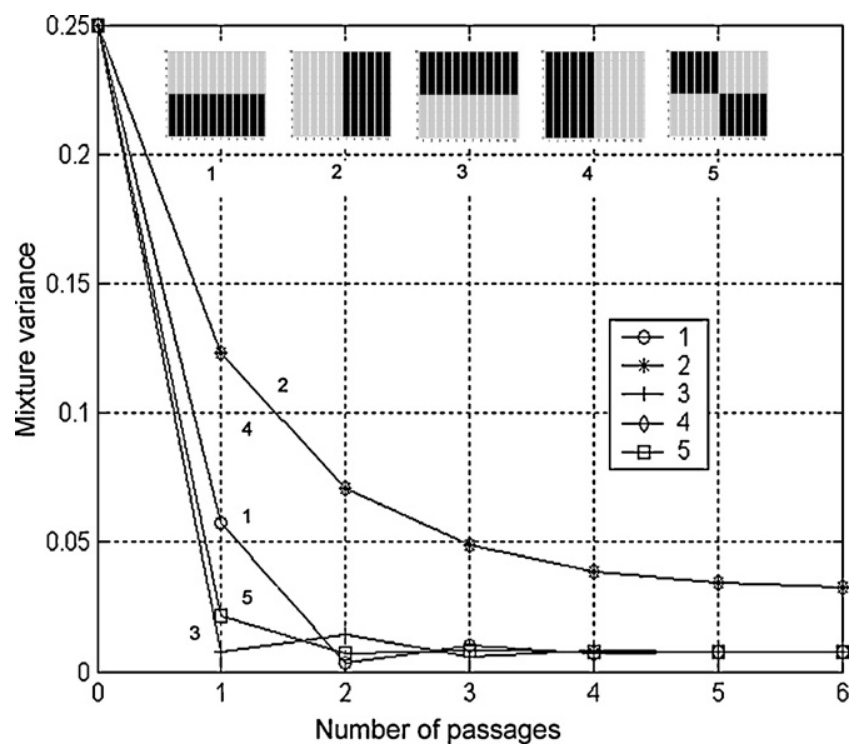

Fig. 14. Influence of the initial configuration on the mixing kinetics ( $A$ is figured in black) for $p_{\mathrm{SA}}=0.25, p_{\mathrm{SB}}=0.2$, and $p_{\mathrm{CA}}=p_{\mathrm{CB}}=0.1$. apparent where horizontal flow is reduced. In the mixing element design close attention must therefore be paid to promoting horizontal motion of the particles, especially in the case where the initial disposition of the components is vertical, as is practically always the case in continuous mixing.

Other initial distributions of the components, but with the same initial intensity of segregation, were considered in the simulation and are reported in Fig. 10a and b. They both led to earlier achievement of the mixture, within just a couple of revolutions of the mixer. However, even if the initial variance is the same, the scale of segregation ("the maximum size of segregated regions", see [20]) is much smaller in these cases. In other words, a large-scale previous displacement of blocks of particles is significant in improving the mixing kinetics.

\subsection{Potentially segregating mixture}

Most of the time, it is necessary to mix components with different physical properties, and probably different flow behaviours. Such mixtures have a segregation potential that is difficult to predict as it will depend on various intermingled factors: particle attraction or rejection, relative sizes, differences in shape, differences in particle size distribution, composition of the mixture and so forth. Sometimes, these factors are combined and may counterbalance their own effects. In the context of the present model, we will consider only binary mixtures and transcribe their differences in terms of the transition probabilities of the model: $p_{\mathrm{sA}}, p_{\mathrm{sB}}, p_{\mathrm{cA}}$ and $p_{\mathrm{cB}}$.

Let us first consider a vertical initial distribution of material (see Fig. 11) and assume the following transition probabilities: $p_{S A}=0.1$, $p_{\mathrm{CA}}=0.5, p_{\mathrm{SB}}=0.1$, and $p_{\mathrm{CB}}=0.3$. It can be said that component $\mathrm{A}$ transits faster in the horizontal direction than component $\mathrm{B}$, and experiences the same quick downwards transitions (low values of $p_{\mathrm{s}}$ ). Fig. 9a can be used to discriminate both products from the viewpoint of their "in-process" flow behaviour. The distributions of the 

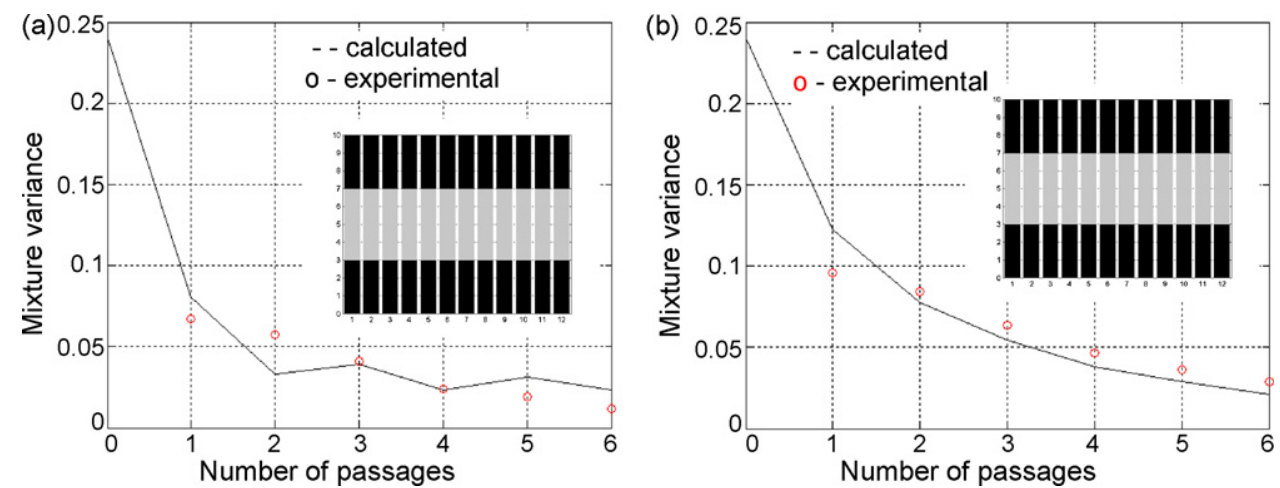

Fig. 15. Model and experimental data comparison for the mixing kinetics obtained in the Sysmix ${ }^{\circledR}$ mixer with (a) or without (b) mixing elements.

components after one and eight passes are also given in the diagrams representing the filling process of the cells. They show that the free surface is actually irregular, due to preferential filling of the right part of the container. This is the result of the different horizontal transition probabilities that favour the retention of $A$ in the vessel.

Fig. $12 \mathrm{a}-\mathrm{c}$ illustrates the influence of the transition probability values on the mixing kinetics by changing alternatively $p_{\mathrm{s}}$ or $p_{c}$ for one of the two components in the case of the initial vertical disposition. A small decrease in the vertical transitions of $A$ by changing $p_{\mathrm{s}}$ from 0.1 to 0.3 leads to a serious slowing down in the process, as well as some oscillations in the variance graph (compare Fig. 12a and b). The mixing kinetics is also improved when $p_{\mathrm{cA}}$ is reduced from 0.5 to 0.4 (compare Fig. 12a and c), which is closer to $p_{\mathrm{cB}}$. This behaviour cannot be explained by the intrinsic horizontal or vertical mobility values as is the case for the non-segregating mixtures. It is more likely to be the result of the differences in transition probabilities between A and B. Indeed, the mixing process is less efficient when the probabilities grow more distant from each other. Fig. 13a-c shows the same ideas in the case of the horizontal arrangement to that concerning the influence of the changing transition probabilities. The differences in the transition probabilities of the two components clearly govern the mixing process. In addition, we can examine the effect of the initial arrangement. As in the non-segregating case, the horizontal disposition improves the mixture quality in terms of mixing kinetics and "final" homogeneity. For example, it is 3-4 times better in terms of variances after two passes in the system than for the previous case. It must also be noted that clearly marked oscillations are now taking place, which must be taken into account in process optimization. In particular, in Fig. 13a, mixture quality is much better after two passes in the mixer than after eight passes.

Fig. 14 shows how the mixing kinetics is influenced by the initial distribution of the components. The probabilities are $p_{\mathrm{SA}}=0.25$, $p_{\mathrm{sB}}=0.2$, and $p_{\mathrm{CA}}=p_{\mathrm{CB}}=0.1$, so that a slight difference exists in the axial or crosswise mobility of particles, component $A$ being retained in the system more than component $B$. It can be seen that the best mixture quality with the smallest mixing time is reached for the distribution in which the component having the higher downward probability (B) is placed at the top of the loading container. Once again, oscillations take place in the case of horizontal arrangements. It is somewhat remarkable to observe that even a decrease in the initial scale of segregation (curve 5) does not lead to a better mixing process.

All these examples of simulations serve to illustrate the sensitivity - in terms of both mixing kinetics and mixing time - of the mixing process to the initial disposition of the component and the relative values of the transition probabilities. Particular attention must be paid to these factors if there is a potential risk of segregation of the particles.

\section{Modelling of the Sysmix ${ }^{\circledR}$ mixer}

To try to validate partially the Markov chain model developed in this work, we have used experimental data obtained and published by Gyenis and Arva in the studies we referred to previously. The authors considered two couples of components in their work, namely quartz-sodium chloride and flour-polypropylene granules, but we will focus only on the first couple. The initial arrangement of the components in the loading container was horizontal and organized in three layers. During the mixing process, the mixer was alternately revolved around the horizontal shaft at the middle height of the mixing zone. The duration of a $180^{\circ}$ rotation was $1.5 \mathrm{~s}$ followed by a standstill of $3.0 \mathrm{~s}$. With different mean particle sizes and densities, the components seemed to segregate during the experiments.

Transition probabilities were matched to the experimental data by minimizing difference between calculated and experimental concentrations in each column of the unloading container at every pass. Fig. 15 reports experimental data and adjusted model results for the Sysmix ${ }^{\circledR}$ mixer with (a) or without (b) mixing elements inserted. The best match was obtained for the following values of the transition probabilities:

- $p_{\mathrm{SA}}=0.02, p_{\mathrm{SB}}=0.3, p_{\mathrm{CA}}=p_{\mathrm{CB}}=0.1$ with mixing elements. - $p_{\mathrm{SA}}=0.01, p_{\mathrm{SB}}=0.12, p_{\mathrm{CA}}=p_{\mathrm{CB}}=0.05$ without mixing element.

The matching result is visually acceptable, which gives a positive impression about the ability of the model to calculate a real case. Some oscillations are found when mixing elements are inserted, but are due to the fact that the transition probability values are closer in this case. This leads us again to conclude that segregation is not only caused by the intrinsic properties of the particles, but is also due to their behaviour in a specific process. As might be imagined, the presence of the mixing elements in the mixer increases the horizontal mobility of the particles, as well as increasing their retention in the system. It helps the mixing kinetics by inducing a quicker decrease in the variance of the mixture, but it also seems that variances are close to each other when the number of passes exceeds 6 . It is therefore probable that the process is not yet optimized from the viewpoint of mixing element design. The present model could be a very valuable tool for this task.

\section{Conclusion}

A two-dimensional model of particulate solid mixing was developed on the basis of Markov chain theory. The prototype was the 
Sysmix ${ }^{\circledR}$ mixer where mixing occurs both in vertical and horizontal directions. The model proposed makes it possible to calculate distribution of components in two directions and to estimate mixture quality accurately. Simulations were run to investigate the effect of initial material loading and to analyse the effect of transition probability values on the mixing kinetics, so as to take particle segregation into account. It was shown that while mixing components that tend to segregate, there is a rational way of loading components, and an optimal number of revolutions giving the maximal mixture quality.

Main issues for improving the current model are:

- Linking transition probabilities to particle flow characteristics in a definite process configuration. This could be done experimentally, but also through the help of other models, like DEM at the level of a cell, for example. This could therefore give rise to a hybrid model of flow in static mixers.

- Validation of the model by new sets of experiments, testing effectively the effects of different process variables on the transition probabilities, and refining the approach by identifying processdependent parameters and product-dependent parameters.

- Optimization of the design and operation of static mixers (number, shape, spatial disposition, type of mixing elements) by using the model. In other words, finding the best combination of transition probabilities and relating this to mixing element characteristics. This constitutes a real technological challenge.

- Considering more realistic features for the Markov model, such as time- or state-dependent transition matrices, or interdependent transition probabilities such as in [21]. The way transition probabilities for a component are affected by the presence of another component of different transition probabilities is still difficult to apprehend.

\section{Appendix A. List of symbols}

\section{$\mathrm{CV} \quad$ coefficient of variation of a mixture}

$c_{i} \quad$ key component content in sample number $i$

$c_{i j} \quad$ content in sample corresponding to the position $i, j$ in the container

$\langle c\rangle \quad$ key component mean content in samples

$i, j \quad$ indexes for matrix elements or for location of samples in the container

$k \quad$ index for transition number

$m, n \quad$ indexes for matrix dimension

$N \quad$ number of samples

$\mathbf{P} \quad$ transition matrix

$\mathbf{P}_{\mathrm{A}}, \mathbf{P}_{\mathrm{B}}$ transition matrices for component $\mathrm{A}$ and $\mathrm{B}$

$\mathbf{P}_{i j} \quad$ block matrix

$p_{i j} \quad$ element of $\mathbf{P}$

$p_{\mathrm{c}} \quad$ transition probability (horizontal)

$p_{\mathrm{cA}}, p_{\mathrm{cB}}$ transition probabilities (horizontal) for component $\mathrm{A}$ and B

$p_{\mathrm{SA}}, p_{\mathrm{SB}} \quad$ transition probabilities (staying in a cell) for component $A$ and $B$

$p_{\mathrm{d}} \quad$ transition probability (downwards)

$p_{\mathrm{s}} \quad$ transition probability (staying in a cell)
$\boldsymbol{S}_{f}^{(k)} \quad$ feed state vector at transition $k$

$\mathbf{S}_{m} \quad$ state matrix

$s_{i} \quad$ element of $\mathbf{S}_{m}$

$s_{i j} \quad$ element of $\mathbf{S}$

$\mathbf{Z} \quad$ zero block matrix

$\sigma \quad$ mixture standard deviation

$\sigma_{x} \quad$ mixture standard deviation in horizontal direction

$\sigma_{z} \quad$ mixture standard deviation in vertical direction

\section{References}

[1] M. Moakher, T. Shinbrot, F.J. Muzzio, Experimentally validated computations of flow, mixing and segregation of non-cohesive grains in 3D tumbling blenders, Powder Technology 109 (2000) 58-71.

[2] J.J. Mc Carthy, J.M. Ottino, Particle dynamics simulation: a hybrid technique applied to granular mixing, Powder Technology 97 (1998) 91-99.

[3] J.Doucet, N. Hudon, F. Bertrand, C. Chaouki, Modeling of the mixing of monodisperse particles using a stationary DEM-based Markov process, Computers \& Chemical Engineering 32 (6) (2008) 1334-1341.

[4] S.J. Chen, L.T. Fan, C.A. Watson, The mixing of solids particles in a motionless mixer-a stochastic approach, AIChE Journal 18 (5) (1972) 984-989.

[5] R.H. Wang, L.T. Fan, Axial mixing of grains in a motionless Sulzer (Koch) mixer, Industrial \& Engineering Chemistry: Process Design \& Development 15 (3) (1976) 381-388.

[6] R.H. Wang, L.T. Fan, Stochastic modelling of segregation in a motionless mixer, Chemical Engineering Science 32 (1977) 695-701.

[7] R.O. Fox, L.T. Fan, Stochastic analysis of axial solids mixing in a fluidized bed, in: 1st World Congress on Particle Technology, vol. III, Nuremberg, 1986, p. 581.

[8] H.G. Dehling, A.C. Hoffman, H.W. Stuut, Stochastic models for transport in a fluidised bed, SIAM Journal of Applied Mathematics 60 (1) (1999) 337-358.

[9] L.T. Fan, S.H. Shin, Stochastic diffusion model of non-ideal mixing in a horizontal drum mixer, Chemical Engineering Science 34 (1979) 811-820.

[10] M. Aoun Habbache, M. Aoun, H. Berthiaux, V. Mizonov, An experimental method and a Markov chain model to describe axial and radial mixing in a hoop mixer, Powder Technology 128 (2002) 150-167.

[11] K. Marikh, H. Berthiaux, V. Mizonov, E. Barantseva, D. Ponomarev, Flow analysis and Markov chain modelling to quantify the agitation effect in a continuous powder mixer, Chemical Engineering Research and Design, Transactions of the IChemE Part A 84 (A11) (2006) 1059-1074

[12] D Ponomarev, C. Gatumel, V. Mizonov, H. Berthiaux, E. Barantseva, Markov chain modelling and experimental investigation of mixing kinetics in static revolving powder mixers, Chemical Engineering and Processing: Process Intensification 48 (3) (2009) 828-836.

[13] J. Gyenis, J. Arva, Mixing mechanism of solids in alternately revolving mixers, I, Powder Handling \& Processing 1 (3) (1989) 247-254.

[14] J. Gyenis, J. Arva, Improvement of mixing rate of solids by motionless mixer grids in alternately revolving mixers, Powder Handling \& Processing 1 (2) (1989) 165-171.

[15] J. Gyenis, F. Katai, Determination and randomness in mixing of particulate solids, Chemical Engineering Science 45 (9) (1990) 2843-2855.

[16] J. Gyenis, J. Arva, J. Nemeth, Mixing and demixing of non ideal solid particle systems in alternating batch mixer, Hungarian Journal of Industrial Chemistry 19 (1) (1991) 69-74.

[17] H. Berthiaux, V. Mizonov, Application of Markov chains in particulate process engineering: a review, The Canadian Journal of Chemical Engineering 82 (6) (2004) 1143-1168.

[18] V. Mizonov, H. Berthiaux, V. Zhukov, S. Bernotat, Application of multidimensional Markov chains to model kinetics of grinding with internal classification, International Journal of Mineral Processing 74 (1001) (2004) 307-315.

[19] H. Berthiaux, V. Mizonov, Application of the theory of Markov chains to model different processes in particle technology, Powder Technology 157 (2005) 128-137.

[20] P.V. Danckwerts, Theory of mixtures and mixing, Research (London) 6 (1953) 355-361.

[21] C.H. Chou, J.R. Johnson, E.G. Rippie, Polydisperse particulate solids mixing and segregation: nonstationary Markov chains, Journal of Pharmaceutical Sciences 66 (1) (1977) 104-106. 